Article

\title{
Fabrication of Functional Polyurethane/Rare Earth Nanocomposite Membranes by Electrospinning and Its VOCs Absorption Capacity from Air
}

\author{
Jun Cong Ge and Nag Jung Choi * \\ Division of Mechanical Design Engineering, Chonbuk National University, 567 Baekje-daero, \\ Jeonjusi 561-756, Jeollabuk-do, Korea; freedefeng@naver.com \\ * Correspondence njchoi@jbnu.ac.kr; Tel.: +82-63-270-4765 \\ Academic Editor: Stephen Ralph \\ Received: 30 December 2016; Accepted: 7 March 2017; Published: 11 March 2017
}

\begin{abstract}
Volatile organic compounds (VOCs) are a source of air pollution and are harmful to both human health and the environment. In this study, we fabricated polyurethane/rare earth (PU/RE) composite nanofibrous membranes via electrospinning with the aim of removing VOCs from air. The morphological structure of PU/RE nanofibrous mats was investigated using field emission scanning electron microscopy (FE-SEM), fourier transform infrared spectroscopy (FTIR), and X-ray diffraction (XRD) experimental analyses. A certain amount of RE (up to $50 \mathrm{wt}$. \% compared to PU pellets) nanoparticles (NPs) could be loaded on/into PU fibers. The tensile strength of PU/RE nanofibrous membranes decreased slightly with the increasing RE powder content. The PU nanofiber containing $50 \mathrm{wt}$. \% RE powder had the smallest fiber diameter of $356 \mathrm{~nm}$; it also showed the highest VOC absorption capacity compared with other composite membranes, having an absorption capacity about three times greater than pure PU nanofibers. In addition, all of the PU/RE nanofibrous membranes readily absorbed styrene the most, followed by xylene, toluene, benzene and chloroform. Therefore, the PU/RE nanofibrous membrane can play an important role in removing VOCs from the air, and its development prospects are impressive because they are emerging materials.
\end{abstract}

Keywords: electrospinning; polyurethane; rare earth; nanocomposites; VOCs

\section{Introduction}

Volatile organic compounds (VOCs) are numerous, varied, and ubiquitous, and they are one of the sources of air pollution. VOCs can be categorized according to their chemical structures: alkanes, aromatic hydrocarbons, esters, aldehydes, alcohols, ethers, and amides [1]. There are about 300 different kinds of VOCs; most day-to-day activities lead to the production of VOCs, such as driving, cooking, building, decorating, painting, and even face-painting [2-4]. A high concentration of VOCs can easily lead to acute poisoning, lightheadedness, headache, dizziness, coughing, nausea, vomiting, and liver poisoning, and can even induce a coma. Benzene and formaldehyde are the most common carcinogenic and highly toxic pollutants in VOCs and they can quickly damage the human respiratory system [1]. Therefore, it is important to effectively reduce the content of VOCs in the air, and finding a way to control and reduce the content of VOCs in air has attracted attention for a long time.

One area of interest includes the development of both VOC detection and control sensor technology [5-9], using the large surface area and large pore volume, among other advantages, of activated carbon to adsorb the VOCs [10-15]. Activated carbon is the most common adsorption method currently used. However, using activated carbon as an adsorption medium is less economic than that of composite nanofibrous materials. Furthermore, the adsorption capacity of the activated carbon decreases with high pressure drops over the adsorbent bed or after initial carbon fouling. 
In general, activated carbon regeneration is very difficult, except when using specialized methods (steam desorption) [16]. On the other hand, there has also been research regarding the development of new composite materials (such as nanocomposite thin films produced via electrospinning) to absorb VOCs [17-20]. Socholten et al. [16] and Kim et al. [19,21] reported that adsorption and desorption of VOCs by electrospun nanofibrous membranes was faster than that of conventional activated carbon.

Electrospinning is a fiber production method that provides a straightforward yet versatile approach for the convenient preparation of continuous fibers [22-25]. It has been widely applied to various fields, such as artificial skin, bandages, bulletproof clothing, battery electrolytes, sensors, and hierarchically structured fibrous composites [26-29]. The composite nanofibrous materials, which were fabricated using electrospinning technology, are more cost-effective, easy to use and versatile for the removal of VOCs [19-21].

Rare earth (RE) materials, 'the Vitamins of Modern Industry', play an important role in industrial development. Smelting and separation of RE raw materials can be accomplished and used for energetic materials, luminescent materials, grinding materials, environmental protection materials, and permanent magnetic materials [30]. In recent years, $\mathrm{RE}$ has been an emerging material for the removal of VOCs, and it contains a series of metal oxides, such as $\mathrm{Ce}_{2} \mathrm{O}_{3}, \mathrm{CeO}_{2}, \mathrm{La}_{2} \mathrm{O}_{3}, \mathrm{Pr}_{2} \mathrm{O}_{3}, \mathrm{Nd}_{2} \mathrm{O}_{3}$, $\mathrm{Sm}_{2} \mathrm{O}_{3}, \mathrm{Er}_{2} \mathrm{O}_{3}$ and so on. Some of these oxides are widely used in glassmaking, catalysts, ceramics and metallurgy [31]. More importantly, many researchers have reported that some of the individual rare earth elements can curb VOC pollution [32-35]. However, the RE elements combined with other materials, especially the nanocomposite fibrous membranes produced via electrospinning, still have not been studied on the removal characteristics of the VOCs.

Therefore, in this study, we investigated the combined characteristics of RE and polyurethane (PU) and their VOC adsorption capacity using the electrospinning technique. The morphology and components and mechanical properties were thoroughly investigated. A certain amount (up to $50 \mathrm{wt}$ \% compared to PU pellets) of RE nanoparticles (NPs) could be loaded on/into PU fibers. The PU nanofiber containing 50 wt. \% RE powder had the highest VOC absorption capacity, about three times greater than that of pure PU nanofibers. The as-fabricated PU/RE nanofibrous membranes are promising facile, practical materials for the removal of VOCs from air in our environment.

\section{Experimental Details}

\subsection{Materials}

Polyurethane (PU) pellets (Estane ${ }^{\circledR}$ Skythane ${ }^{\circledR}$ X595A-11) were purchased from Lubrizol Advanced Materials, Inc., Cleveland, OH, USA, and were used as the polymeric matrix. RE powder were purchased from ZIBO WEIJIE RARE EARTH CO., LTD, Zibo, China. $N$, $N$-Dimethylformamide (DMF) was purchased from Showa Chemical Co., Ltd., Tokyo, Japan. Extra pure methyl ethyl ketone (MEK, 2-butanone) was purchased from Junsei Chemical Co., Ltd, Tokyo, Japan. In VOCs absorption experiments, five different kinds of gases (styrene, xylene, chloroform, benzene, and toluene) (purity $99.9 \%$, AR grade) were analyzed.

\subsection{Fabrication of Composite Nanofibrous Membranes}

The fabrication process of PU/RE nanofibrous membranes is given as follows. First, the PU pellets were dried (constant temperature: $80^{\circ} \mathrm{C}$ ) for about $3 \mathrm{~h}$ in a dry oven before dissolving them in the solvent. Second, $10 \mathrm{wt}$ \% \% PU pellets were dissolved in a DMF/MEK (50:50 by weight) mixing solution, using a magnetic stirrer dissolved for $12 \mathrm{~h}$ at room temperature. Then $0,10,30$, and $50 \mathrm{wt}$. \% (compared to PU pellets) RE powders were added into above the solution as electrospun precursors via ultrasonication for $2 \mathrm{~h}$. The process of electrospinning is shown in Figure 1. The electrospun temperature and relative humidity are constantly controlled at $23 \pm 2{ }^{\circ} \mathrm{C}$ and $40 \%-50 \%$, respectively. All PU/RE spinning solutions were electrospun at $15 \mathrm{kV}$ of high-voltage electricity with an $18 \mathrm{~cm}$ tip-to-collector distance, the rotating speed of the rotating collector was $650 \mathrm{rpm}$, and the solution 
feed rate was $1 \mathrm{~mL} / \mathrm{h}$ [19-21]. After electrospinning, the composite PU/RE nanofibrous membranes were dried (constant temperature: $80{ }^{\circ} \mathrm{C}$ ) for $12 \mathrm{~h}$ in the vacuum oven. The PU nanofibers containing 0, 10 wt. \%, 30 wt. \% and 50 wt. \% of RE powder are denoted as PU, PU/RE-10, PU/RE-30 and PU/RE-50, respectively.

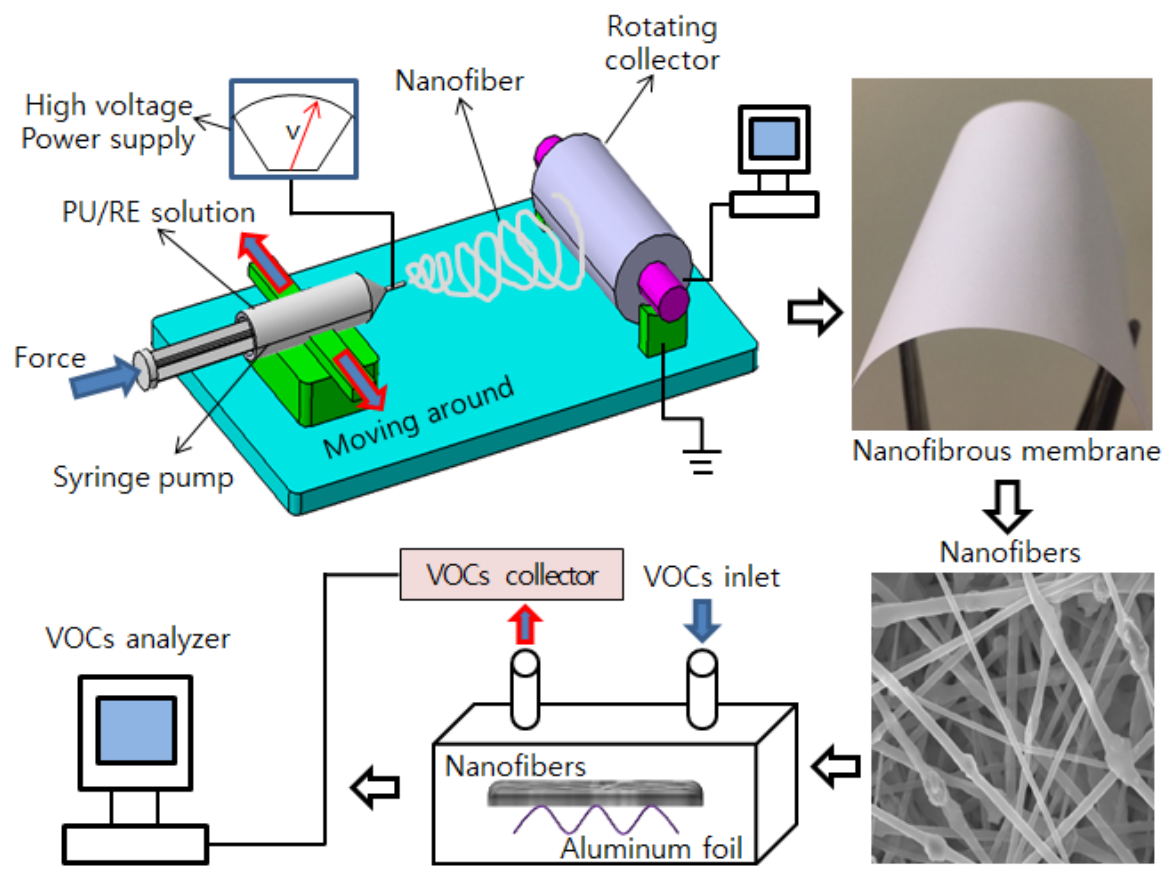

Figure 1. Schematic diagram of the electrospinning setup and volatile organic compounds (VOCs) absorption experiment.

\subsection{Characterization}

The morphology of the PU/RE nanofibrous membranes was observed using field emission scanning electron microscopy (FE-SEM, JIB-4601F, JEOL Ltd., Tokyo, Japan). The viscosity and conductivity of the spinning solutions were measured by an electric conductivity meter (EC meter cm-40G, Hyogo, Japan) and a viscometer (Brookfield LVDH-II) at room temperature. $\mathrm{N}_{2}$ adsorption-desorption isotherms were examined at $77 \mathrm{k}$ by an ASAP 2020 physisorption analyzer (Micromeritics Co., Norcross, GA, USA). The mechanical properties of the PU/RE nanofibrous membranes were measured by a universal testing machine. Fiber diameters were measured with the ImageJ software on 50 random filaments. The Fourier transform infrared (FT-IR, Spectrum GX, PerkinElmer, Lnc., San Francisco, CA, USA) spectra of as-prepared PU/RE nanofibrous membranes were obtained using a Paragon 1000 spectrometer. An X-ray diffraction (XRD) analysis was carried out using a Multipurpose High Performance $X$-ray Diffractometer ( $X^{\prime}$ pert Powder, PANalytical, Eindhoven, The Netherlands).

\subsection{VOCs Absorption Experiment}

The ability of the as-obtained PU/RE nanofibrous membranes to absorb VOCs was tested in a closed container with an air inlet and outlet, as shown in Figure 1. Pristine PU and PU/RE nanofibrous membranes were cut into $8 \mathrm{~cm} \times 8 \mathrm{~cm}$ sections, respectively. A rotary pump was used to remove impurities and other contaminants on the fiber surface and container. Firstly, VOCs were thoroughly mixed by each component of $100 \mu \mathrm{g}$ involving styrene, xylene, chloroform, benzene, and toluene. Next, methanol was used to dilute the solution and to prepare $2 \mu \mathrm{g}$ of VOCs. Finally, a syringe filled with $2 \mu \mathrm{g}$ of VOCs was injected into a container that contained PU/RE nanofibrous membranes. In order to make the surface of the nanofibrous membranes more fully contact with VOCs, padded aluminum foil 
was placed under the nanofibrous membranes as a scaffold. Then the VOCs were absorbed fully by the composite PU/RE nanofibrous membranes for $1 \mathrm{~h}$ at room temperature. Afterwards, a nitrogen stream was used to feed the unabsorbed VOCs into a Tenax absorber (Tenax-GR; Japan Analytical Industry, Tokyo, Japan). In this VOCs absorption experiment, the VOCs absorption ability of PU/RE nanofibrous membranes was analyzed by GC/MS (gas chromatography-mass spectroscopy, XEVO TQ-S, Waters Corporation, Milford, CT, USA), Automated Purge \& Trap analyzer JTD-505II (Japan Analytical Industry Co., Ltd., Tokyo, Japan), and AGC/MS QP 2010 Plus (Shimadzu, Kyoto, Japan). More specific VOCs analysis methods have been previously shown [18,21].

\section{Results and Discussion}

\subsection{Physical Properties of Spinning Solutions and Properties of PU/RE Fiber}

The electrospinning effect and VOC adsorption capacity of the PU/RE nanofibrous membranes were determined by the physical properties of the spinning solutions and the properties of the PU/RE composite nanofibers. Table 1 shows the physical properties of the spinning solutions and the properties of the PU/RE composite nanofibers. The specific surface area was measured by the $\mathrm{N}_{2}$ adsorption-desorption isotherms test via the Brunauer-Emmett-Teller (BET) method. It was found that the viscosity, conductivity and specific surface area increased by increasing the amount of RE powder in the spinning solution. However, the fiber diameter first increased from $489 \mathrm{~nm}$ to $524 \mathrm{~nm}$ and then decreased from $524 \mathrm{~nm}$ to $356 \mathrm{~nm}$ when the relative content of the RE powder reached $50 \mathrm{wt}$. \%. The PU nanofiber containing $50 \mathrm{wt}$. \% of the RE powder had the smallest fiber diameter, which was $356 \mathrm{~nm}$. The high conductivity of the spinning solution $(0.315 \mathrm{~ms} / \mathrm{m})$ led to a better electrospinning effect [21].

Table 1. Physical properties of polyurethane/rare earth (PU/RE) spinning solutions.

\begin{tabular}{lccccc}
\hline \multirow{2}{*}{$\begin{array}{l}\text { Spinning } \\
\text { Solutions (PU/RE) }\end{array}$} & Viscosity (cps) & \multirow{2}{*}{$\begin{array}{l}\text { Conductivity } \\
(\mathbf{m s} / \mathbf{m})\end{array}$} & \multicolumn{2}{c}{ Fiber Diameter $(\mathbf{n m})$} & \multirow{2}{*}{$\begin{array}{c}\text { BET Surface } \\
\text { Area }\left(\mathbf{m}^{2} / \mathbf{g}\right)\end{array}$} \\
\cline { 4 - 5 } & & 0.172 & $227-877$ & 489 & 6.853 \\
PU & 379.0 & 0.183 & $357-966$ & 513 & 7.147 \\
PU/RE-10 & 439.0 & 0.191 & $262-871$ & 524 & 7.729 \\
PU/RE-30 & 464.2 & 0.315 & $179-591$ & 356 & 11.207 \\
PU/RE-50 & 504.6 & Dean & \\
\hline
\end{tabular}

BET: Brunauer-Emmett-Teller; PU: PU nanofibers containing 0 wt. \% RE powder; PU/RE-10: PU nanofibers containing $10 \mathrm{wt}$. \% RE powder; PU/RE-30: PU nanofibers containing $30 \mathrm{wt}$. \% RE powder; PU/RE-50: PU nanofibers containing 50 wt. \% RE powder.

\subsection{Morphological Characteristics of the PU/RE Nanofibrous Membranes}

In order to analyze the pristine RE powder, FE-SEM and energy-dispersive X-ray spectroscopy (EDX) images of the pristine RE powder were taken before introducing the nanofibers, which are shown in Figure 2. It can be seen from Figure 2a that the pristine RE powder has very irregular shapes and different sizes. Figure $2 b$ shows the composition and amounts of each element in the pristine RE powder. There were many rare elements in the pristine RE powder, including $\mathrm{Ce}, \mathrm{Pr}, \mathrm{Nd}, \mathrm{Ho}, \mathrm{Tm}, \mathrm{Sm}$, Dy, and an extremely small amount of Pm.

Figure 3 shows the morphology and the fiber diameter distribution of the composite PU/RE nanofibrous membranes. From Figure $3 a-d$, it was observed that the changes of the fiber morphology and fiber diameter distribution correlated very closely with the increasing amounts of RE powder in the PU fibers. The pristine PU fibers had smooth surfaces and an average fiber diameter of $489 \mathrm{~nm}$. However, it was found that there were a lot of RE NPs embedded in the PU fibers, and the number of RE NPs increased with an increased concentration of RE powder (Figure 3b-d). In addition, some particle agglomeration existed in Figure $3 d$ because higher concentrations of RE powder cannot be completely dispersed into the spinning solution. During the actual experimental operation, the electrospinning nozzle was blocked two times when the PU/RE nanofibrous membrane with 50 wt. \% RE was 
produced. We also tested other concentrations of PU/RE spinning solutions (PU/RE-55 and PU/RE-60) using the same electrospinning conditions. In these cases, the clogging of the electrospinning nozzle was very serious, and the composite PU/RE nanofibrous membranes could not be manufactured when the higher concentration of RE was added into the PU solution.

Figure 3e-h show the average fiber diameters of the PU, PU / RE-10, PU / RE-30 and PU / RE-50 nanofibrous membranes, which were 489, 513, 524 and $356 \mathrm{~nm}$, respectively. Each of the reported $\mathrm{PU} / \mathrm{RE}$ nanofibers' diameters represents an average of 50 random fibers. The fiber diameters increased slightly as the amount of RE powder increased from 0 to $30 \mathrm{wt}$. \%, and then showed a marked decrease when the relative amount of RE powder was $50 \mathrm{wt}$. \%. The PU/RE-50 possessed the smallest average diameter of $356 \mathrm{~nm}$. A probable cause of this may be due to the higher conductivity of the PU/RE-50 spinning solution compared to the other spinning solutions. Based on [21], this result has also been confirmed, as shown in Figure 3e-h. The fiber diameter distribution range of PU/RE-50 nanofibrous membrane was more narrow and concentrated compared with other nanofibrous membranes, and most of them were distributed in the range of $300-500 \mathrm{~nm}$.

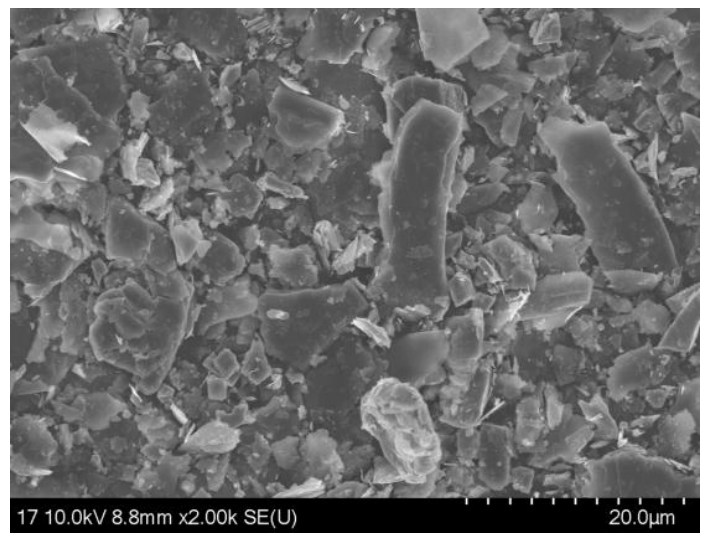

(a)

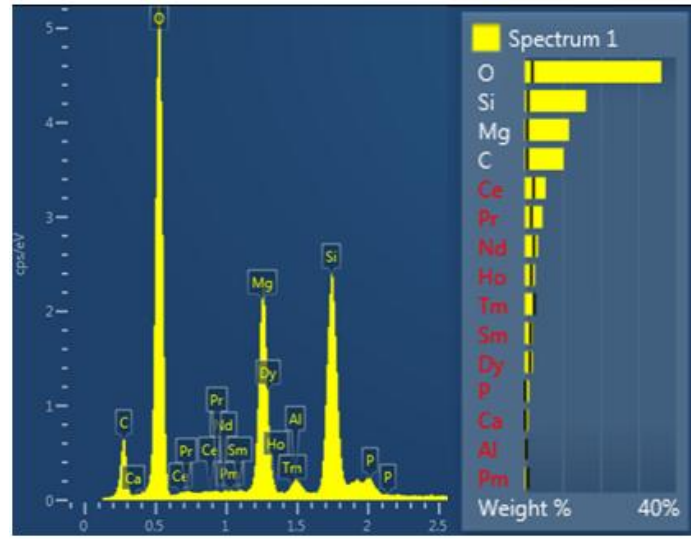

(b)

Figure 2. Field emission scanning electron microscopy (FE-SEM) (a) and energy-dispersive X-ray spectroscopy (EDX) (b) images of the pristine RE powder.
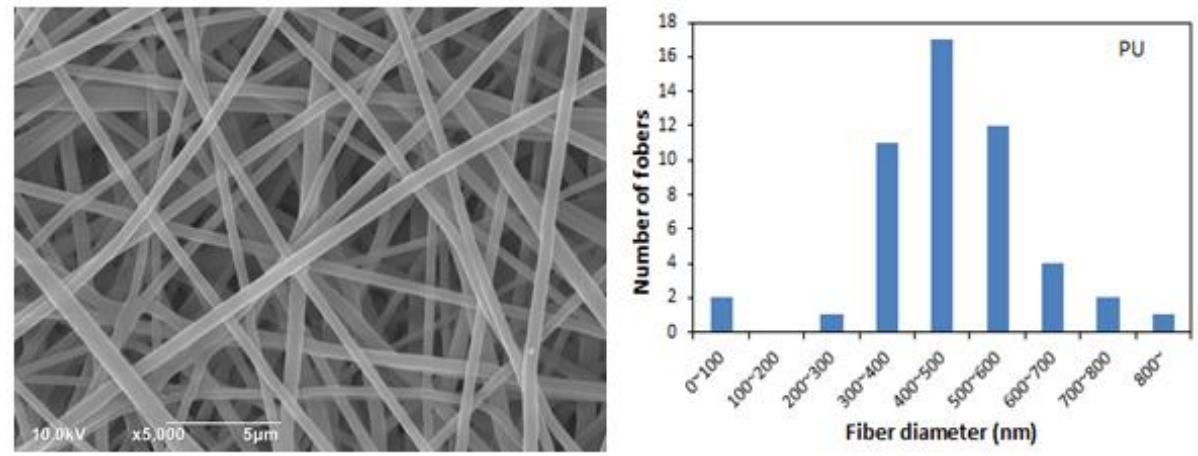

(a)

Figure 3. Cont. 

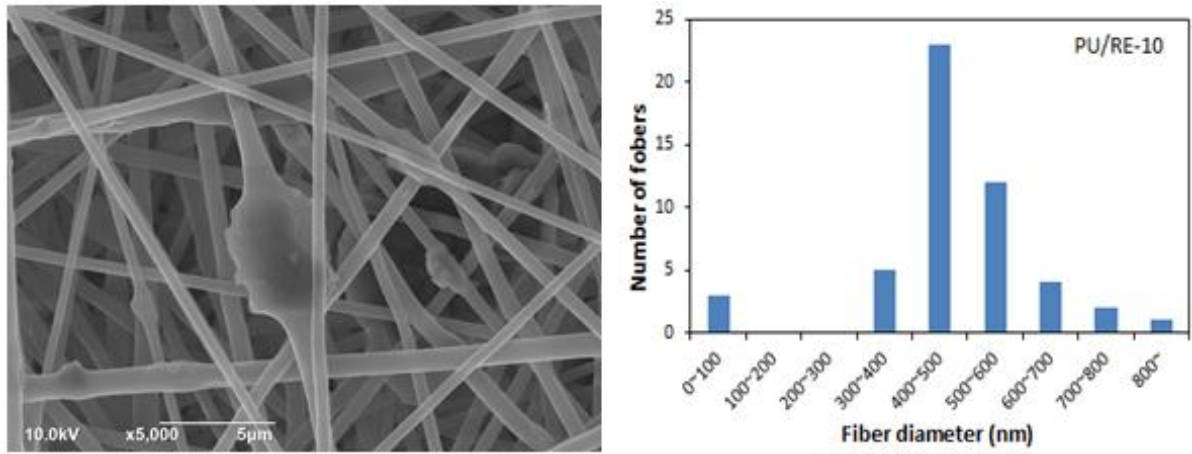

(b)
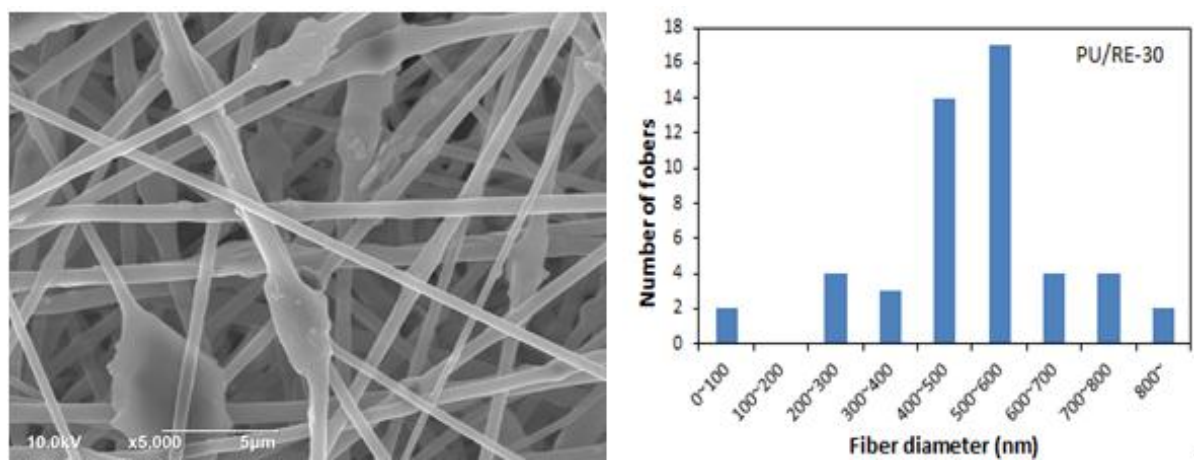

(c)
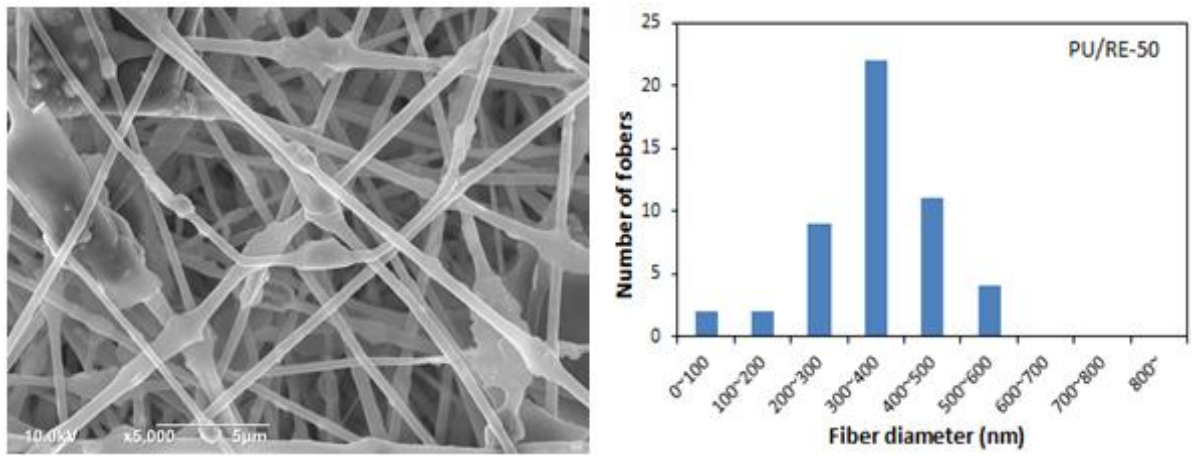

(d)

Figure 3. FE-SEM images and diameter distributions of (a) polyurethane (PU); (b) PU/RE-10; (c) PU/RE-30 and (d) PU/RE-50.

\subsection{Morphological Characteristics of the PU/RE Nanofibrous Membranes}

Nanofibrous filtration membranes exhibit efficient VOC absorption efficiency as well as good mechanical properties, traits which may indicate a potential for commercial application, such as in an air filter installed in a vehicle that can reduce the vehicle's fuel consumption due to its lighter weight. The mechanical properties of the PU/RE nanofibrous membranes are presented in Figure 4. The tensile strength of each of the composite PU/RE nanofibrous membranes was tested five times to obtain an average value. As shown in Figure 4, the tensile strengths of the PU/RE nanofibrous membranes decreased gradually as the amount of RE powder increased, from 10.63 to $8.28 \mathrm{MPa}$. The tensile strength of the pristine PU nanofibrous membrane was $10.63 \mathrm{MPa}$, and it is similar to the research results of Kim et al. [21]. The tensile strength of the PU/RE nanofibrous membranes decreased by only $22.1 \%$ when the amount of RE powder reached $50 \mathrm{wt}$. \% compared with the pristine PU nanofibrous membrane. An explanation may be that adding the RE powder to the PU spinning solution led to slight disordering of the fibers as well as the destruction of fibrous regularity during electrospinning. 
From Figure $3 b-d$, an obvious disorder of the fibers can be seen as the amount of RE powder in the PU fibers increases. However, while even these disorders and agglomerated particles can decrease the tensile strength of the composite PU/RE nanofibrous membranes, the composite PU/RE nanofibrous membranes can still be fully used as an air filter because their tensile strength is above 8.28 $\mathrm{MPa}[36,37]$. On the other hand, the PU/RE nanofibrous membranes have outstanding mechanical properties and more than a $700 \%$ strain.

In order to further analyze the RE NPs embedded into the PU fiber and the interactions between the RE powder and PU, the XRD patterns and FT-IR spectra of the PU/RE nanofibrous membranes were investigated. The XRD patterns of the PU nanofibers containing different amounts of RE powder (0 wt. \%, 10 wt. \%, 30 wt. \% and 50 wt. \%) are shown in Figure 5. Originally, the RE powder exhibited many characteristic peaks, but after the introduction of RE powder into the PU nanofiber, most of the main XRD peaks emerged and the peak intensity of the composite PU/RE nanofibrous membranes increased significantly when increasing the amount of the RE powder in the PU fibers. It was observed that the RE powder was sufficiently incorporated into the PU NPs in the electrospinning process, and the RE powders were effectively embedded into the PU fibers, which was consistent with the FEM-SEM results. In addition, with the characteristics of the XRD pattern peaks, all the above results demonstrated that a large amount of $\mathrm{MgSiO}_{3}$ and $\mathrm{CePO}_{4}$ existed in the as-prepared PU/RE fiber mats. The crystal phases of $\mathrm{PrPO}_{4}, \mathrm{Mg}_{0.5} \mathrm{Ce}_{2}\left(\mathrm{PO}_{4}\right)_{3}, \mathrm{Mg}_{3} \mathrm{Si}_{4} \mathrm{O}_{10}(\mathrm{OH})_{2}$ were also found in the composite $\mathrm{PU} / \mathrm{RE}$ nanofibrous membranes, which indicated that they are a main constituent component of $\mathrm{Si}^{4+}$, $\mathrm{Mg}^{2+}, \mathrm{Ce}^{3+}, \mathrm{Pr}^{3+}$ and $\mathrm{Nd}^{3+}$ and they have been successfully incorporated into the host lattice [38].

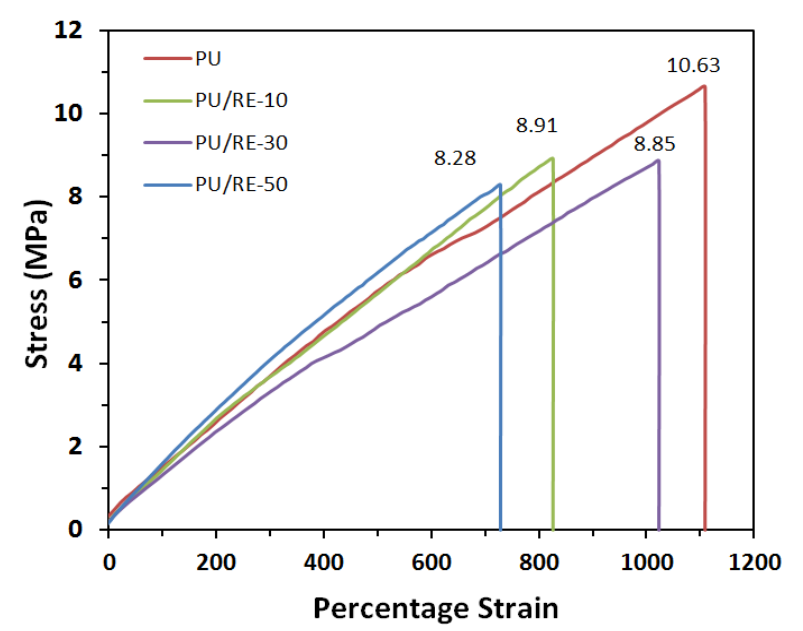

Figure 4. Tensile strength of electrospun PU mats with different amounts of RE.

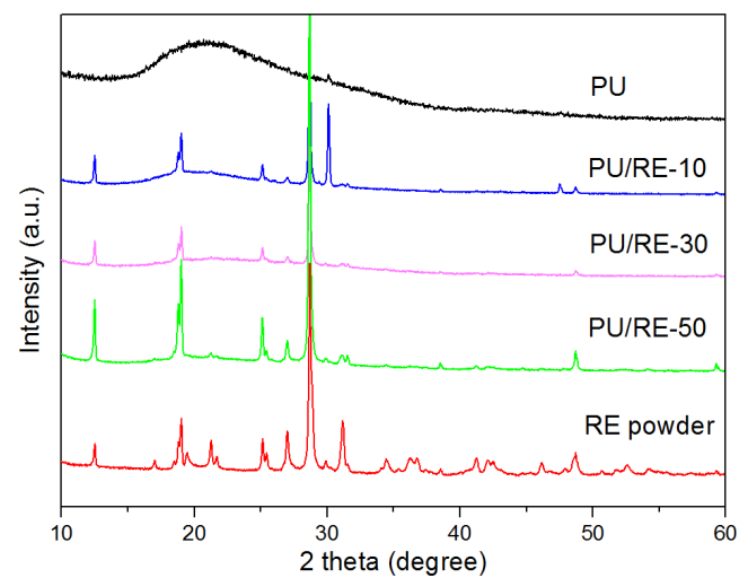

Figure 5. X-ray diffraction (XRD) patterns of different PU/RE nanofibrous mats. 
In order to further observe the incorporation characteristics of the RE powder and PU and the phase formation process, the FTIR spectra of the PU nanofibers containing different amounts of RE powder (0 wt. \%, 10 wt. \%, 30 wt. \%, 50 wt. \%) are presented in Figure 6. As shown in Figure 6, the FTIR spectrums of the PU/RE composite fibers have no significant changes from 1774 to $2820 \mathrm{~cm}^{-1}$. The FTIR spectrum of PU is characterized by the $\mathrm{C}=\mathrm{O}$ vibration and the $\mathrm{N}-\mathrm{H}$ vibration at $1726 \mathrm{~cm}^{-1}$ and $1700 \mathrm{~cm}^{-1}$, respectively. The strong band around $3675 \mathrm{~cm}^{-1}$ is associated with the Si-OH bond asymmetric stretching vibrations, and that around $1016 \mathrm{~cm}^{-1}$ is the $\mathrm{Si}-\mathrm{O}$ stretching vibrations, and the peaks (around $3675 \mathrm{~cm}^{-1}$ and $1016 \mathrm{~cm}^{-1}$ ) of the composite PU/RE nanofiber gradually increased by increasing the amount of RE powder in the PU fiber. In addition, the band at $1072 \mathrm{~cm}^{-1}$ represents the C-O-C vibration $[39,40]$. The changes were apparent by increasing the amount of RE powder in the PU fiber, which indicates that the addition of the RE powder affected the $\mathrm{C}-\mathrm{O}-\mathrm{C}$ vibration in the PU fiber, showing a weak interaction between the molecules in the RE powder and the PU. This result further shows that the RE powder completely combined with the PU during the electrospinning process.

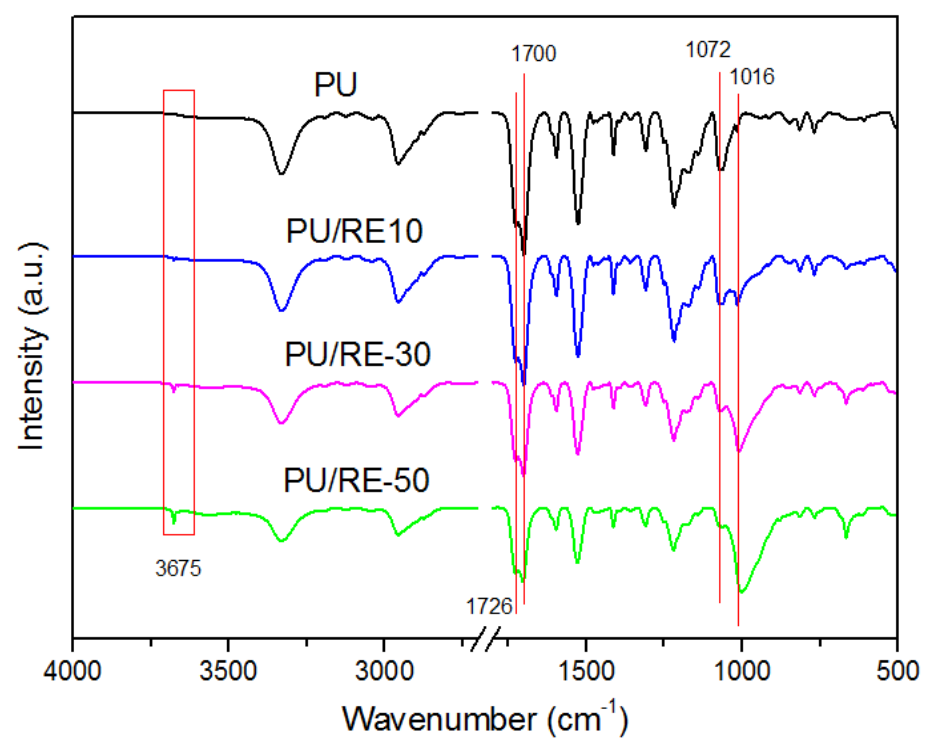

Figure 6. FTIR spectra of different PU/RE nanofibrous mats.

\subsection{Absorption Characteristics of the Composite PU/RE Nanofibrous Membranes}

The VOC absorption characteristics of the PU nanofibers containing different amounts of RE powder (0 wt. \%, 10 wt. \%, 30 wt. \%, 50 wt. \%) are shown in Figure 7. As shown in Figure 7, it can be seen that the pure PU nanofibrous membrane had a weak VOC absorption capacity (about 10\%), while the pure RE powder had a better VOC absorption capacity compared with the pure PU nanofibrous membrane. However, the RE powder will limit its usability. To integrate the merit and eliminate the defect of both PU and RE, the RE-incorporated PU fibers were electrospun before the VOC absorption experiment. The VOC absorption capacity of the composite PU/RE nanofibrous membranes increased as the amount of RE powder in the PU fibers increased. The PU nanofiber containing 50 wt. \% RE powder had the highest VOC absorption capacity. In addition, the absorption capacity of the PU nanofiber containing 50 wt. \% RE powder increased by 320\%, 210\%, 208\% and 187\% compared with that of the pure PU nanofibrous membrane during the absorption of styrene, xylene, toluene, benzene and chloroform, respectively. This may be due to the decreasing fiber diameter of the PU/RE composite fibers as the amount of RE powder increased, which subsequently increased the surface area of the composite fiber, producing a high physical absorption capacity. Furthermore, the higher BET surface area of the PU nanofiber containing $50 \mathrm{wt}$ \% RE powder is 1.64 times greater than the pure PU nanofiber, as shown in Table 1, and it plays an important role in reducing the VOCs [21]. On the other hand, in the chemical adsorption capacity, the RE elements have a certain VOC absorption capacity 
that has been reported by other researchers [36-39]. In combination with the physical absorption capacity and the chemical adsorption capacity of the PU/RE nanofibrous membranes, the PU/RE-50 had the highest VOC absorption capacity.

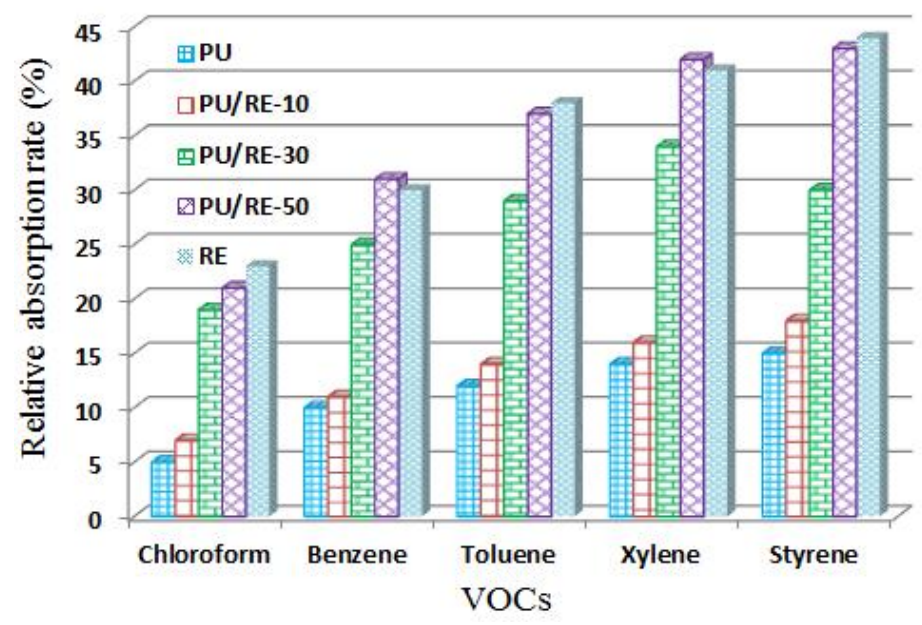

Figure 7. VOC absorption capacity of different nanofibrous mats.

As shown in Figure 7, it can be also seen that the composite PU/RE nanofibrous membranes most easily absorbed styrene, followed by xylene, toluene, benzene and chloroform. Minot et al. [34] and Kim et al. [21] also reported the same result and they suggested that the VOC absorption capacity of the fiber is related to the formation of $\pi$-complexes on the fiber surface. The VOC absorption capacity of the fiber becomes stronger as more $\pi$-complexes are formed on the fiber surface. On the other hand, the presence of a methyl group would increase the stability of the $\pi$-complex [34]. The chemical formulas of styrene, xylene, toluene, benzene and chloroform are $\mathrm{C}_{8} \mathrm{H}_{8}, \mathrm{C}_{8} \mathrm{H}_{10}, \mathrm{C}_{7} \mathrm{H}_{8}, \mathrm{C}_{6} \mathrm{H}_{6}$ and $\mathrm{CHCl}_{3}$, respectively. From these chemical formulas it can be seen that styrene contains the most methyl groups, followed by xylene, toluene, benzene and chloroform (no methyl group). Hence, the VOC absorption capacity of the composite PU/RE nanofibrous membranes is the strongest for the absorption of styrene compared with the other VOCs (xylene, toluene, benzene and chloroform).

\section{Conclusions}

In summary, a series of PU nanofibers containing different amounts of RE powder $(0 \mathrm{wt} . \%$, $10 \mathrm{wt}$. \%, $30 \mathrm{wt}$. \%, $50 \mathrm{wt}$. \%) with a high VOC absorption capacity can be successfully produced via electrospinning. At RE powder concentrations greater than $50 \mathrm{wt}$ \% , however, the electrospinning nozzle will be seriously blocked. The PU/RE-50 fiber had the smallest fiber diameter, with an average fiber diameter of $356 \mathrm{~nm}$. In addition, this fiber also had outstanding mechanical properties, with an average tensile strength of $8.28 \mathrm{MPa}$ and a percentage strain that was above $700 \%$. Most importantly, it had the highest VOC absorption capacity, more than three times that of the pure PU nanofiber. In addition, the composite PU/RE nanofibrous membranes most easily absorbed styrene, followed by xylene, toluene, benzene and chloroform. This work verified that the PU/RE nanofibrous membranes have a high VOC absorption capacity, and our report also has a certain reference value regarding the study of composite functional nanofibers.

Acknowledgments: This research was supported by the Basic Science Research Program through the National Research Foundation of Korea (NRF) funded by the Ministry of Education (Project No. 2016R1D1A1B03931616). The authors thank Jeong Hyeon Kim from the Division of Mechanical Design Engineering of Chonbuk National University for his help in performing this experiment. The authors also thank PH.D. Yanan Liu and PH.D. Yang Liu from the Department of BIN Convergence Technology of Chonbuk National University for their help in development of the paper. 
Author Contributions: All authors contributed equally to this work. All authors designed the experimental apparatus, analyzed the data, discussed the results and implications and commented on the manuscript at all stages. Jun Cong Ge and Nag Jung Choi conceived and designed the experiments and analyzed the data; Jun Cong Ge performed the experiments and wrote the paper; Nag Jung Choi contributed reagents and materials.

Conflicts of Interest: The authors declare no conflict of interest.

\section{References}

1. Kawamura, K.; Vestergaard, M.; Ishiyama, M.; Nagatani, N.; Hashiba, T.; Tamiya, E. Development of a novel hand-held toluene gas sensor: Possible use in the prevention and control of sick building syndrome. Measurement 2006, 39, 490-496. [CrossRef]

2. Mirzaei, A.; Leonardi, S.G.; Neri, G. Detection of hazardous volatile organic compounds (VOCs) by metal oxide nanostructures-based gas sensors: A review. Ceram. Int. 2016, 42, 15119-15141. [CrossRef]

3. Bartzis, J.; Wolkoff, P.; Stranger, M.; Efthimiou, G.; Tolis, E.I.; Maes, F.; Nørgaard, A.W.; Ventura, G.; Kalimeri, K.K.; Goelen, E.; et al. On organic emissions testing from indoor consumer products' use. J. Hazard. Mater. 2015, 285, 37-45. [CrossRef] [PubMed]

4. Mendell, M.J. Indoor residential chemical emissions as risk factors for respiratory and allergic effects in children: A review. Indoor Air 2007, 17, 259-277. [CrossRef] [PubMed]

5. Temel, F.; Tabakci, M. Calix[4]arene coated QCM sensors for detection of VOC emissions: Methylene chloride sensing studies. Talanta 2016, 15, 221-227. [CrossRef] [PubMed]

6. Lee, S.W.; Takahara, N.; Korposh, S.; Yang, D.H.; Toko, K.; Kunitake, T. Nanoassembled thin film gas sensors. III. Sensitive detection of amine odors using $\mathrm{TiO}_{2} /$ poly (acrylic acid) ultrathin film quartz crystal microbalance sensors. Anal. Chem. 2010, 82, 2228-2236. [CrossRef] [PubMed]

7. Fan, X.; Du, B. Selective detection of trace $p$-xylene by polymer-coated QCM sensors. Sens. Actuators B 2012, 166, 753-760. [CrossRef]

8. Yoo, H.Y.; Bruckenstein, S. A novel quartz crystal microbalance gas sensor based on porous film coatings. A high sensitivity porous poly(methylmethacrylate) water vapor sensor. Anal. Chim. Acta 2013, 785, 98-103. [CrossRef] [PubMed]

9. Hyodo, T.; Hashimoto, T.; Ueda, T.; Nakagoe, O.; Kamada, K.; Sasahara, T.; Tanabe, S.; Shimizu, Y. Adsorption/combustion-type VOC sensors employing mesoporous $\gamma$-alumina co-loaded with noble-metal and oxide. Sens. Actuators B 2015, 220, 1091-1104. [CrossRef]

10. Sundarrajan, S.; Tan, K.L.; Lim, S.H.; Ramakrishna, S. Electrospun Nanofibers for Air Filtration Applications. Procedia Eng. 2014, 75, 159-163. [CrossRef]

11. Fletcher, A.J.; Yüzak, Y.; Thomas, K.M. Adsorption and desorption kinetics for hydrophilic and hydrophobic vapors on activated carbon. Carbon 2006, 44, 989-1004. [CrossRef]

12. Ramos, M.E.; Bonelli, P.R.; Cukierman, A.L.; Ribeiro Carrott, M.M.L.; Carrott, P.J.M. Adsorption of volatile organic compounds onto activated carbon cloths derived from a novel regenerated cellulosic precursor. J. Hazard. Mater. 2010, 177, 175-182. [CrossRef] [PubMed]

13. Oh, G.Y.; Ju, Y.W.; Kim, M.Y.; Jung, H.R.; Kim, H.J.; Lee, W.J. Adsorption of toluene on carbon nanofibers prepared by electrospinning. Sci. Total Environ. 2008, 393, 341-347. [CrossRef] [PubMed]

14. Wang, H.; Zhu, T.; Fan, X.; Na, H. Adsorption and desorption of small molecule volatile organic compounds over carbide-derived carbon. Carbon 2014, 67, 712-720. [CrossRef]

15. Bai, Y.; Huang, Z.H.; Wang, M.X.; Kang, F. Adsorption of benzene and ethanol on activated carbon nanofibers prepared by electrospinning. Adsorption 2013, 19, 1035-1043. [CrossRef]

16. Scholten, E.; Bromberg, L.; Rutledge, G.C.; Hatton, T.A. Electrospun Polyurethane Fibers for Absorption of Volatile Organic Compounds from Air. ACS Appl. Mater. Interfaces 2011, 3, 3902-3909. [CrossRef] [PubMed]

17. Cui, P.Q.; Zhou, H.G.; Li, C.; Wu, S.P.; Xiao, Y. Characteristics of using layered double hydroxides to reduce the VOCs from bituminous materials. Constr. Build. Mater. 2016, 123, 69-77. [CrossRef]

18. Gil, R.R.; Ruiz, B.; Lozano, M.S.; Martín, M.J.; Fuent, E. VOCs removal by adsorption onto activated carbons from biocollagenic wastes of vegetable tanning. Chem. Eng. J. 2014, 245, 80-88. [CrossRef]

19. Kim, H.J.; Pant, H.R.; Choi, N.J.; Kim, C.S. Composite electrospun fly ash/polyurethane fibers for absorption of volatile organic compounds from air. Chem. Eng. J. 2013, 230, 244-250. [CrossRef]

20. Ge, J.C.; Kim, J.H.; Choi, N.J. Electrospun polyurethane/loess powder hybrids and their absorption of volatile organic compounds. Adv. Mater. Sci. Eng. 2016, 2016, 8521259. [CrossRef] 
21. Kim, H.J.; Pant, H.R.; Choi, N.J.; Kim, C.S. Fly Ash/Polyurethane Thin Film for the Adsorption of Volatile Organic Compounds (VOCs) from Air. Fibers Polym. 2014, 15, 1393-1398. [CrossRef]

22. Purwar, R.; Goutham, K.S.; Srivastava, C.M. Electrospun Sericin/PVA/Clay nanofibrous mats for antimicrobial air filtration mask. Fibers Polym. 2016, 17, 1206-1216. [CrossRef]

23. Sun, G.; Sun, L.; Xie, H.; Liu, J. Electrospinning of nanofibers for energy applications. Nanomaterials 2016, 6, 129. [CrossRef]

24. Ercolano, G.; Farina, F.; Cavaliere, S.; Jones, D.J.; Rozière, J. Nickel based electrospun materials with tuned morphology and composition. Nanomaterials 2016, 6, 236. [CrossRef]

25. Liu, Y.; Park, M.; Ding, B.; Kim, J.; El-Newehy, M.; Al-Deyab, S.S.; Kim, H.Y. Facile electrospun polyacrylonitrile/poly(acrylic acid) nanofibrous membranes for high efficiency particulate air filtration. Fibers Polym. 2015, 16, 629-633. [CrossRef]

26. Rohatgi, C.V.; Dutta, N.K.; Choudhury, N.R. Separator membrane from crosslinked poly(Vinyl Alcohol) and poly(methyl vinyl ether-alt-maleic anhydride). Nanomaterials 2015, 5, 398-414. [CrossRef]

27. Kim, H.J.; Pant, H.R.; Park, C.H.; Tijing, L.D.; Choi, N.J.; Kim, C.S. Hydrothermal growth of mop-brush-shaped $\mathrm{ZnO}$ rods on the surface of electrospun nylon-6 nanofibers. Ceram. Int. 2013, 39, 3095-3102. [CrossRef]

28. Kumbar, S.G.; Nukavarapu, S.P.; James, R.; Nair, L.S.; Laurencin, C.T. Electrospun poly(lactic acid-co-glycolic acid) scaffolds for skin tissue engineering. Biomaterials 2008, 29, 4100-4107. [CrossRef] [PubMed]

29. Kim, S.J.; Kang, Y.J.; Choi, N.J. Optical-fiber electronic speckle pattern interferometry for quantitative measurement of defects on aluminum liners in composite pressure vessels. J. Opt. Soc. Korea 2013, 17, 50-56. [CrossRef]

30. Naumov, A.V. Review of the world market of rare-earth metals. Russ. J. Non-Ferr. Met. 2008, 49, 14-22.

31. Charalampides, G.; Vatalis, K.I.; Apostoplos, B.; Ploutarch-Nikolas, B. Rare earth elements: Industrial applications and economic dependency of europe. Procedia Econ. Financ. 2015, 24, 126-135. [CrossRef]

32. Guo, H.; Xue, B.; Chen, M. Catalytic oxidation of VOCs over the structured bimetallic catalyst $0.1 \% \mathrm{Pt}-0.75 \%$ $\mathrm{CeO}_{2}$ /SSWM. Sustain. Environ. Res. 2015, 25, 167-170.

33. Carabineiro, S.; Konsolakis, M.; Marnellos, G.; Asad, M.; Soares, O.; Tavares, P.; Pereira, M.; Melo Órfão, J.; Figueiredo, J. Ethyl acetate abatement on copper catalysts supported on ceria doped with rare earth oxides. Molecules 2016, 21, 644. [CrossRef] [PubMed]

34. Konsolakis, M.; Carabineiro, S.A.C.; Marnellos, G.E.; Asad, M.F.; Soares, O.S.G.P.; Pereira, M.F.R.; Órfão, J.J.M.; Figueiredo, J.L. Volatile organic compounds abatement over copper-based catalysts: Effect of support. Inorg. Chim. Acta 2017, 455, 473-482. [CrossRef]

35. Yang, L.; Tao, D.; Yang, X.; Li, Y.; Guo, Y. Synthesis, characterization, and antibacterial activities of some rare Earth metal complexes of pipemidic acid. Chem. Pharm. Bull. 2003, 51, 494. [CrossRef] [PubMed]

36. Liu, B.; Zhang, S.; Wang, X.; Yu, J.; Ding, B. Efficient and reusable polyamide-56 nanofiber/nets membrane with bimodal structures for air filtration. J. Colloid Interface Sci. 2015, 457, 203-211. [CrossRef] [PubMed]

37. Gopal, R.; Kaur, S.; Ma, Z.; Chan, C.; Ramakrishna, S.; Matsuura, T. Electrospun nanofibrous filtration membrane. J. Membr. Sci. 2006, 281, 581-586. [CrossRef]

38. Wang, Z.; Lou, S.; Li, P. Enhanced orange-red emission of $\mathrm{Sr}_{3} \mathrm{La}\left(\mathrm{PO}_{4}\right)_{3}: \mathrm{Ce}^{3+}, \mathrm{Mn}^{2+}$ via energy transfer. J. Lumin. 2014, 156, 87-90. [CrossRef]

39. Li, Q.; Pang, J.; Wang, B.; Tao, D.; Xu, X.; Sun, L.; Zhai, J. Preparation, characterization and microwave absorption properties of barium-ferrite-coated fly-ash cenospheres. Adv. Powder Technol. 2013, 24, 288-294. [CrossRef]

40. Iwasiki, M.; Shinjoh, $\mathrm{H}$. Analysis of the adsorption state and desorption kinetics of $\mathrm{NO}_{2}$ over Fe-zeolite catalyst by FT-IR and temperature-programmed desorption. Phys. Chem. Chem. Phys. 2010, 12, 2365-2372. [CrossRef] [PubMed]

(C) 2017 by the authors. Licensee MDPI, Basel, Switzerland. This article is an open access article distributed under the terms and conditions of the Creative Commons Attribution (CC BY) license (http:/ / creativecommons.org/licenses/by/4.0/). 